\title{
Helical Computed Tomography - Anatomy of the Cat Abdomen
}

\author{
E. GAVRILAS ${ }^{1 *}$, F. MIRO ${ }^{2}$, B. BLANCO ${ }^{3}$, R. LUCENA ${ }^{3}$, P.J. GINEL ${ }^{3}$, M. NOVALES ${ }^{3}$ \\ ${ }^{1}$ Department of Semiology and Medical Pathology, Faculty of Veterinary Medicine, USAMV, Iasi, Romania \\ ${ }^{2}$ Department of Anatomy and Comparative Pathology, Faculty of Veterinary Medicine, University of \\ Cordoba, Cordoba, Spain \\ ${ }^{3}$ Department of Animal Medicine and Surgery, Faculty of Veterinary Medicine, University of Cordoba, \\ Spain \\ *Corresponding author: elena.gavrilas.dmv@gmail.com
}

Bulletin UASVM Veterinary Medicine 73(1) / 2016,

Print ISSN 1843-5270; Electronic ISSN 1843-5378

DOI:10.15835/buasvmcn-vm: 11937

\begin{abstract}
Computed tomography (CT) is a most valuable technique for the diagnosis of abdominal disorders. In veterinary medicine, $\mathrm{x}$-ray and ultrasound are the imaging modalities of choice for the abdomen, but CT can provide information that cannot be obtained by other means. One mature, female, domestic cat, $4 \mathrm{~kg}$ weight was used for this study. The cat was clinically healthy, with no abnormal findings at hematological and biochemical analysis. It was premedicated with $20 \mu \mathrm{g} / \mathrm{kg}$ medetomidine and $0.3 \mathrm{mg} / \mathrm{kg}$ butorphanol IM. For induction of anesthesia, 3 to $5 \mathrm{mg} / \mathrm{kg}$ propofol was administered IV. Anesthesia was maintained with a mixture of isoflurane and oxygen. The patient was positioned in sternal recumbency. CT scans were performed with a helical CT scanner Hi Speed CT/e Dual; General Electric. Technical factors were $120 \mathrm{kVp}$ and $50 \mathrm{~mA}$ settings, with 1 rotation/s. All sections were imaged with soft-tissue window. CT scans were performed after IV injection of iodinated contrast medium, iopamidol, $2 \mathrm{~mL} / \mathrm{kg}$. The images are presented in progression from the 8th thoracic vertebra to the level of the pelvic inlet. Identifiable anatomical structures are labelled on the CT images. The stomach is left-sided with the gastric folds less numerous and thinner than those in the dogs. The normal small intestine contains less gas in cats. The feline cecum is very short. The liver showed a low attenuation with the mediastinum-vascular window settings, allowing better visualisation of the hepatic lobes and interlobular fissures. The gallbladder and bile ducts were also better visualised. CT imaging studies can provide additional information in patients with radiographic and ultrasonographic patterns of abdominal disorders, as a specialised imaging method, a useful preoperative technique and a precise staging tool. CT improves the image quality further and allows better timing of vascular phases. Helical CT angiography enables visualization of contrast-enhanced abdominal vessels using only a peripheral venous injection of iodinated contrast medium.
\end{abstract}

Keywords: abdomen, anatomy, cat, computed tomography

\section{INTRODUCTION}

Computed tomography (CT) is a most valuable technique for the diagnosis of abdominal disorders, and in human medicine has become the imaging modality of choice for the abdomen (El Sherif et al., 1999; Novelline et al., 1999). In veterinary medicine, $\mathrm{x}$-ray and ultrasound are the imaging modalities of choice for the abdomen, although, it is increasingly recognized that CT can provide information that cannot be obtained by other means. Helical or spiral CT is an important recent development offering a number of imaging advantages in evaluating thoracic and abdominal diseases (Samii et al., 1998).

\section{MATERIALS AND METHODS}

One mature, female, domestic short-haired cat, weighing $4 \mathrm{~kg}$, was used for this study. The 
cat was clinically healthy, presenting no abnormal findings on physical examination and routine haematological and biochemical analysis. The cat was not fed for $12 \mathrm{~h}$ before imaging, but had free access to water. It was premedicated with a combination of $20 \mu \mathrm{g} / \mathrm{kg}$ medetomidine and $0.3 \mathrm{mg} / \mathrm{kg}$ butorphanol IM (Torbugesic, Zoetis). For induction of anaesthesia, 3 to $5 \mathrm{mg} / \mathrm{kg}$ propofol (Propofol Lipuro 1\%, Braun) was administered intravenously. Anaesthesia was maintained after intubation with a mixture of isoflurane and oxygen. Rapidly breathing patient was hiperventilated in order to obtein an apnea period for the imaging acquisition. The cat was monitored during the entire procedure.

The patient was positioned in sternal recumbency.

CT scans were performed with a helical CT scanner (CT Hi Speed CT/e Dual; General Electric Yokogawa Medical Systems LTD, Hino, Japan). Technical factors were $120 \mathrm{kVp}$ and 50 $\mathrm{mA}$ settings and a scan speed of 1 rotation/s. Transverse slices of $5 \mathrm{~mm}$ thickness and $2.5 \mathrm{~mm}$ image spacing were obtained from the level of the 8th thoracic vertebra and extended caudally to the level of the pelvic inlet. Scans were performed with $5 \mathrm{~mm}$ collimation and a pitch of 1 . All sections were imaged with soft-tissue window settings and the cranial abdomen was also imaged with mediastinumvascular window settings. CT scans were performed after IV injection of iodinated contrast medium, iopamidol (Scanlux, Iberoinvesa Pharma) in dose of $2 \mathrm{~mL} / \mathrm{kg}$. The original CT data were transferred as DICOM images to an image analysis with DICOM viewer (Horos v.1.0.6, 32-bit) to perform image study. The original transverse slices were reformatted into the sagittal and dorsal planes (Caceres et al. 2008).

\section{RESULTS AND DISCUSSION}

The images are presented in progression from the level of the 8th thoracic vertebra to approximately the level of the pelvic inlet. Identifiable anatomical structures are labelled on the CT images. The stomach is left - sided with the pylorus mostly located at the level of the vertebral column, and with the gastric folds less numerous and thinner than those in the dogs (Vazquez et al., 2000). On CT, an intramural lucent band and hypodense layer corresponds to fat in the gastric submucosa. This band is a normal anatomic feature. The normal small intestine contains less gas in cats than in dogs (Vazquez et al., 2000). The feline cecum is very short.The liver showed a low attenuation with the mediastinumvascular window settings, allowing better visualization of the hepatic lobes and interlobular fissures. The gallbladder and bile ducts were also better visualized with this window due to the hypoattenuation. However, the rest of the cranial

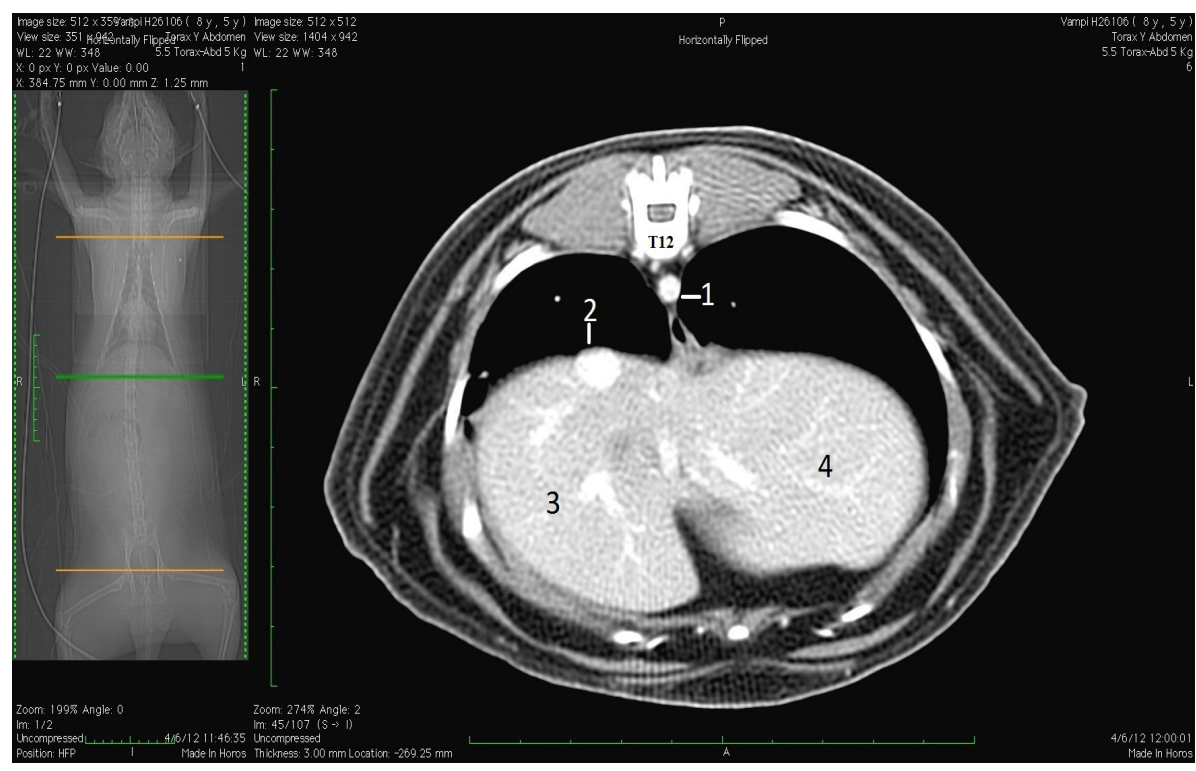

Fig.1. Transverse CT image (cranial 12th thoracic vertebra) of the cranial abdomen after IV contrast administration, soft tissue window. (1) thoracic aorta, (2) caudal vena cava, (3) right medial lobe of liver, (4) left medial lobe of liver. 


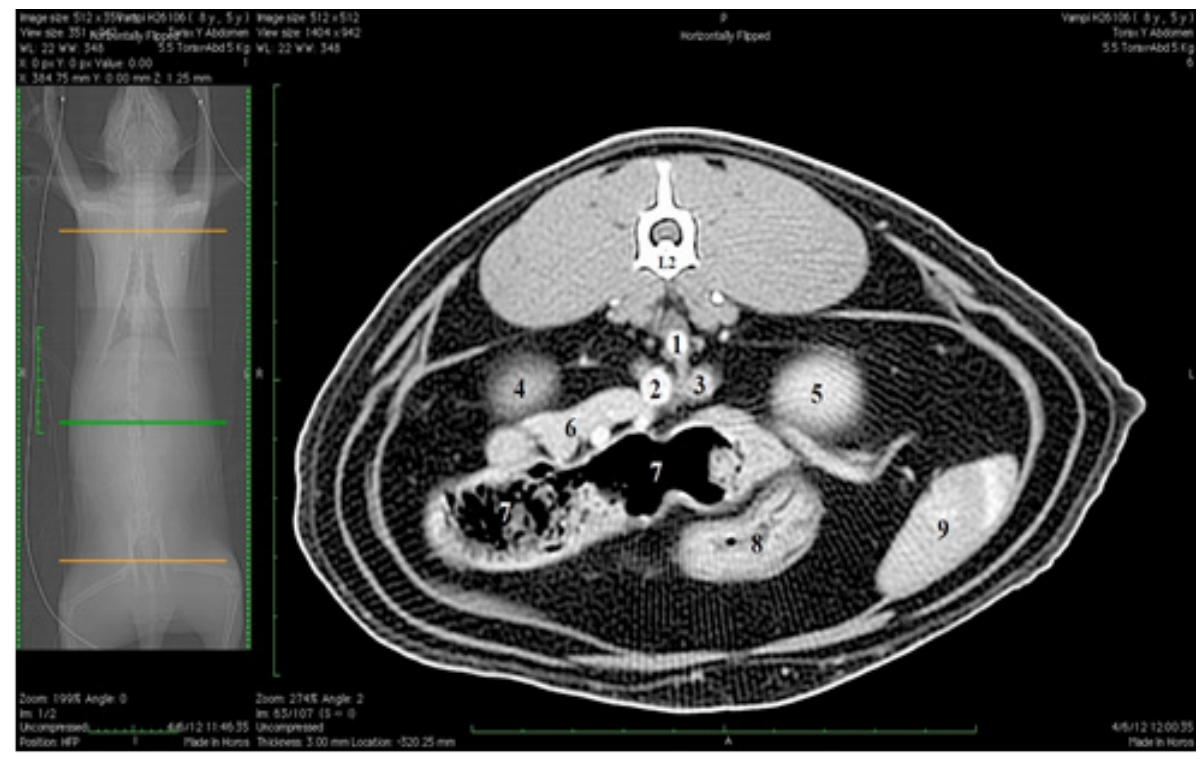

Fig.2. Transverse CT image (medial 2nd lombar vertebra) of the cranial abdomen after IV contrast administration, soft tissue window. (1) abdominal aorta, (2) caudal vena cava, (3) left adrenal gland, (4) right kidney, (5) left kidney, (6) liver, (7) transverse colon, (8) body of stomach, (9) splein.

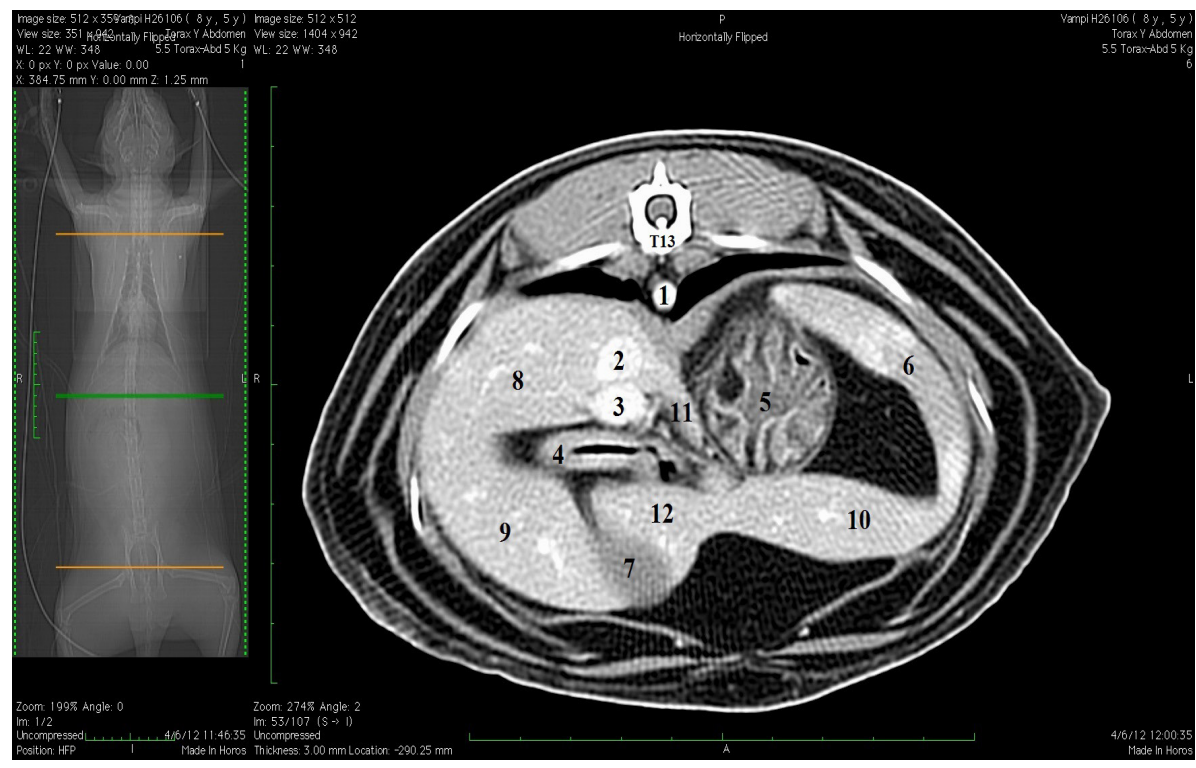

Fig.3. Transverse CT image (caudal 13th thoracic vertebra) of the cranial abdomen after IV contrast administration, soft tissue window. (1) thoracic aorta, (2) caudal vena cava, (3) portal vein, (4) cranial part of duodenum, (5) fundus of stomach, (6) spleen, (7) gallbladder, (8) right lateral lobe of liver, (9) right medial lobe of liver, (10) left lateral lobe of liver, (11) papillary process of the caudate lobe of liver, (12) quadrate lobe of liver.

abdominal structures were well visualized in both window settings (Vignoli et al., 2011).

\section{CONCLUSION}

CT imaging studies can provide additional information in patients with radiographic and ultrasonographic patterns of abdominal disorders, as a specialized imaging method, a useful preoperative technique and a precise staging tool for tumoral disorders. CT improves the image quality further and allows better timing of vascular phases. Helical CT angiography enables visualization of contrast-enhanced abdominal vessels using only a peripheral venous injection of iodinated contrast medium. 


\section{REFERENCES}

1. Caceres A, Zwingenberger A, Aronson L, Mai W (2008). Characterization of Normal Feline Renal Vascular Anatomy with Dual-Phase CT Angiography. Veterinary Radiology \& Ultrasound 49(4):350-356.

2. El Sherif A, Mc Pherson SJ, Dixon AK (1999). Spiral CT of the abdomen: increased diagnostic potential. European Journal of Radiology 31:43-52.

3. Novelline RA, Rhea JT, Rao P (1999). Helical CT in emergency radiology. Radiology 213:321-339.
4. Samii V, Biller D, Koblik P (1998). Normal Cross-Sectional Anatomy of the Feline Thorax and Abdomen: Comparison of Computed Tomography and Cadaver Anatomy. Veterinary Radiology \& Ultrasound 39(6):504-511.

5. Vazquez JM, Ramirez G, Gil F, LaTorre R, Moreno F (2000). Atlas de Anatomia Clinica: Perro y Gato. Cavidades Toracica, Abdominal y Pelviana. AG Novograf, SA, Murcia, Spain.

6. Vignoli M, Saunders J (2011). Gastrointestinal Tract. In: Schwarz T, Saunders J(Eds). Veterinary Computed Tomography, Wiley-Blackwell, 325-330. 